\title{
Marketinginstrument mit Potenzial
}

Sponsoring hat sich im Marketing-Mix der Unternehmen etabliert: Rund 4,4 Milliarden Euro pro Jahr geben deutsche Unternehmen laut der Studie „Sponsor Visions 2012“ für das Kommunikationstool aus - rund $50 \%$ mehr als noch vor zehn Jahren. Auch in der Schweiz liegt Sponsoring im Trend: Mit durchschnittlich $15 \%$ Anteil am Kommunikationsbudget nimmt das Marketinginstrument hinter der klassischen Werbung (28\%) den zweithöchsten Rang ein, wie eine Untersuchung der Universität St. Gallen und der IG Sponsoring aus dem Jahr 2010 zeigt.

Doch gerade aufgrund dieser Entwicklung steht Sponsoring zunehmend unter kritischer Beobachtung in den Unternehmen: „Ist Sponsoring für uns wirklich ein gutes Kommunikationstool?“, „Verkaufen wir über Bandenwerbung mehr Autos, mehr Solarmodule oder Versicherungen?" Die Frage nach der Refinanzierbarkeit von Rechte- und Umsetzungskosten dominiert bereits seit Jahren die Sponsoring-Diskussion. Immer wieder neu entfachen Interessengruppen wie Fachverbände oder Sponsorenvereinigungen sowie Fachzeitschriften die Diskussion über die Effektivität und Effizienz des Kommunikationstools. Selbstverständlich erheben Sponsoring treibende Unternehmen eine große Anzahl an Daten, gewichten diese und verknüpfen sie mit finanziellen Beträgen. Allerdings existieren keine allgemein anerkannten Bewertungsoder Erhebungsrichtlinien, wie es sie beispielsweise in der klassischen Werbung gibt. Insbesondere fehlt ein umfassendes Controlling-System, das einen Vergleich zwischen verschiedenen Kommunikationsmedien und somit Effizienzbeurteilungen erlaubt. Das ist ein Grund, warum viele Sponsoringverantwortliche ihre Engagements oftmals lediglich hinsichtlich Reichweiten und Imagewerten prüfen lassen.

Die Frage nach der Wirksamkeit und der Wirtschaftlichkeit von Sponsoring bildet den Schwerpunkt der vorliegenden Ausgabe der Marketing Review St. Gallen. Zudem widmen sich einige Beiträge weiteren aktuellen Herausforderungen so-

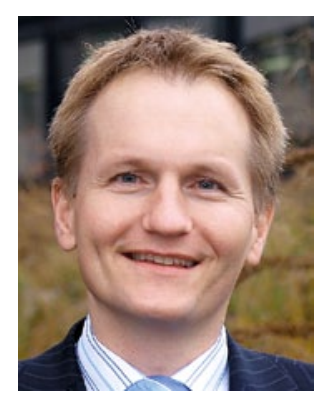

Prof. Dr. Sven Reinecke

Direktor des Instituts für Marketing sowie Leiter des Kompetenzzentrums Marketing Performance Management an der Universität St. Gallen

E-Mail:sven.reinecke@unisg.ch

wie den Erfolgskriterien und Stärken dieses Kommunikationsinstruments.

Im Interview erklären Johannes Seibert, Marketingleiter BMW Deutschland, und Magnus Wiese, Leiter Messen, Events und Sportmarketing BMW Deutschland, wie der Automobilhersteller durch unterschiedliche Sponsoringmaßnahmen neue Zielgruppen erschließen möchte.

Wir freuen uns, mit dieser Ausgabe eine kritische Reflexion anzuregen. Zielgerichtetes Sponsoring erfüllt eine wichtige Rolle im Marketing-Mix. Ohne Wirkungsnachweis und geeignete Steuerungsinstrumente verkommt es jedoch zu einem Luxusinstrument, für das künftig - zu Recht - kaum noch finanzielle Ressourcen bereitgestellt werden dürften.



